\title{
Jain's hand retractor system and stand: an innovative device for hand surgery
}

\author{
Aakansh Jain \\ Department of Plastic Surgery, King George's Medical University, Lucknow, India
}

Solo hand surgery is becoming increasingly common, mostly due to a lack of assistants and dedicated operating theatres. The solo hand surgeon faces challenges of proper hand positioning, elevation, and skin flap/tissue retraction. Most commercially available hand retractors or stands do not address all the relevant problems and are not economically feasible for trainee surgeons. The Jain's hand retractor system and stand, described herein, provides solutions to all these problems, and its simple design helps surgeons to reproduce it easily.

Keywords Device design, medical / Hand / Reconstructive surgery / Innovativeness

\author{
Correspondence: Aakansh Jain \\ Department of Plastic Surgery, King \\ George's Medical University, Room \\ No. 4, Resident Hostel, Lucknow \\ 226003, India \\ Tel: +91-99-9047-9456 \\ E-mail: aakansh.awakes@gmail.com
}

The author would like to thank Ms. Deepa Thapa (Artist, Department of Plastic Surgery, King George's Medical University, Lucknow, India).

Received: March 8, $2021 \bullet$ Revised: May 3, $2021 \bullet$ Accepted: May 27, 2021

pISSN: 2234-6163 • elSSN: 2234-6171 • https://doi.org/10.5999/aps.2021.00409 • Arch Plast Surg 2021;48:389-391

\section{INTRODUCTION}

The increasing number of trauma cases has decreased the operative time and skilled assistance allotted to each surgery. Therefore, most hand surgeons throughout the world are required to perform solo surgery in emergency procedure rooms or as daycare surgical procedures [1]. Hand surgeons face major problems, including hand positioning, retraction of the skin flaps, and the need to hold other fingers away from the operated-upon areas. A few sophisticated commercial self-retaining hand retractor systems are available on the market, such as various lead hands and HandStand (Hand Surgery Stand, Metairie, LA, USA) [2-4], but these systems are expensive, heavy, and not easily available, especially to trainee residents learning to perform hand surgery. Many hand surgeons have created innovations in the past to address this problem with limited success, such as self-retaining hand retractors using fishhooks [5], malleable retractors, and stands $[4,6]$. Furthermore, the commercially available hand retractor systems have some drawbacks that restrict their use; for example, the lead hand and its variations, which are commonly used at many centers, neither provide elevation nor have a provision for the retraction of skin flaps. These instruments also lack the do-it-yourself (DIY) type of freedom. These problems, which we encountered while performing hand surgery, motivated us to devise a solution that can be easily made with the spare materials available in the operating theatre to simultaneously address all the above issues.

\section{IDEA}

Jain's hand retractor system and stand is an ideal hand retractor system in which adjustments can be made according to the surgeon's need. It is made using a 15- to 20-cm-long piece of Kramer wire, three metallic rods, two connectors or clamps, and a 6-inch distractor (Fig. 1). All components are easily available as left-overs from procedures involving orthopedic patients. It can also be made with alternative materials; for example, instead of the Kramer wire piece, a metallic soap stand of similar size or 


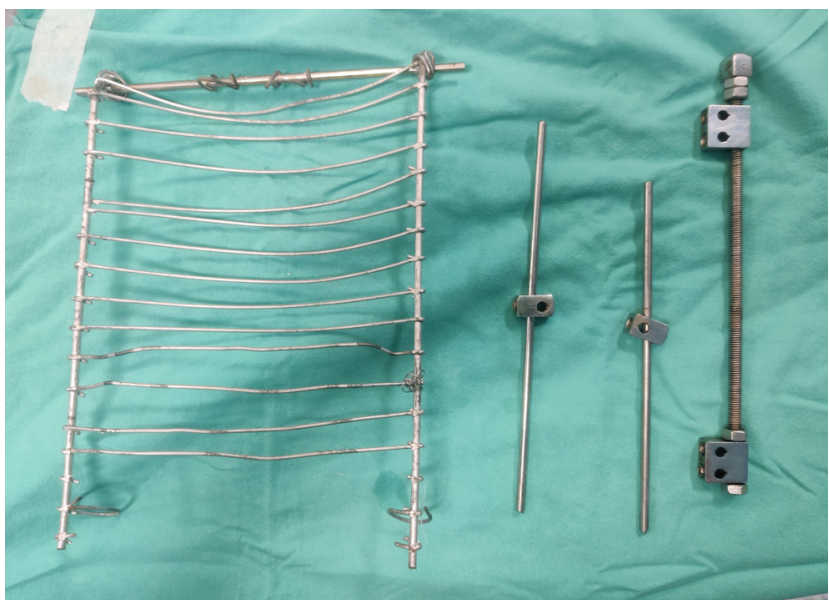

Fig. 1. Components of Jain's hand retractor system and stand (Kramer wire with a bar, 2 metallic bars with a connector, 6-inch distractor).
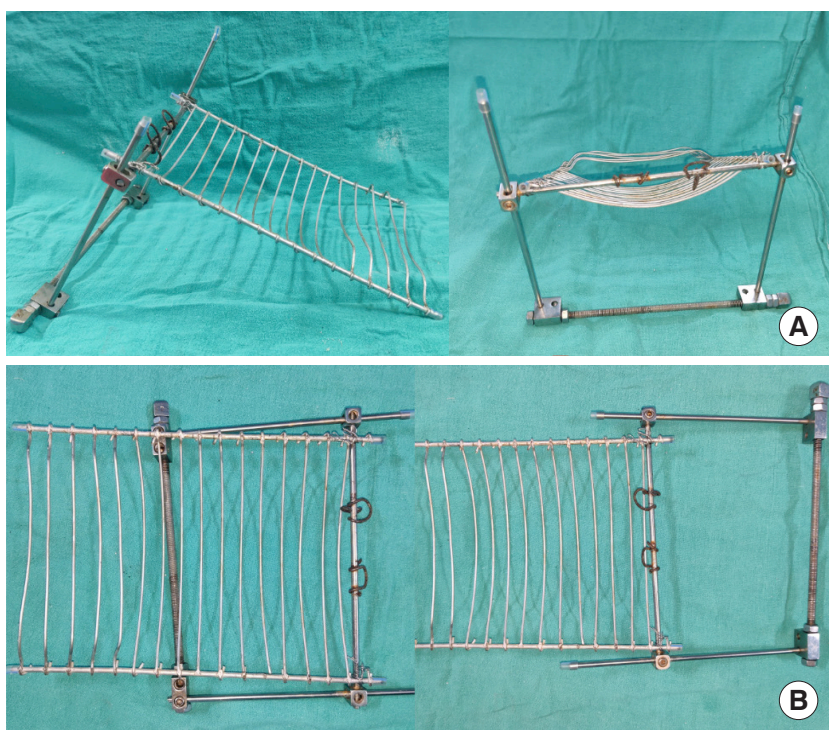

Fig. 2. Assembled Jain's hand retractor system and stand. (A) Standing. (B) Folded.

stainless-steel stand for holding hot objects can be used, and instead of the distractor, another pair of clamps with a connecting bar can be used. The entire assembly barely weighs $150-200 \mathrm{~g}$. These materials can then be assembled as shown in Figs. 2 and 3 to make Jain's hand retractor system and stand.

Existing hand retractor systems, HandStand, and lead hands were devised to help surgeons, and primarily aim to hold other fingers away from the field of work and aid to some extent in the retraction of flaps. Although these devices allay the need for assistants and make the operative field less crowded, they require folded towels or sandbags to provide hand inclination and positioning. Therefore, the system as a whole ultimately becomes messy and bulky. Cases of trauma require thorough washing and

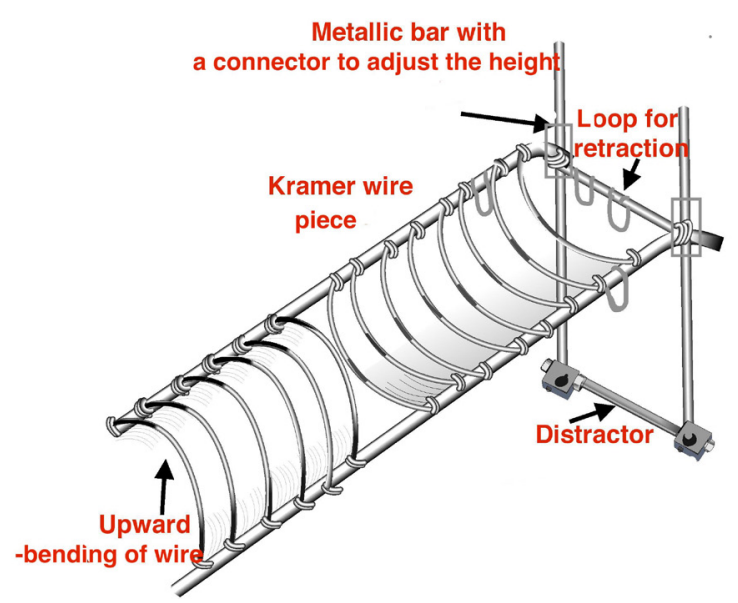

Fig. 3. Line diagram of components of Jain's hand retractor system and stand.
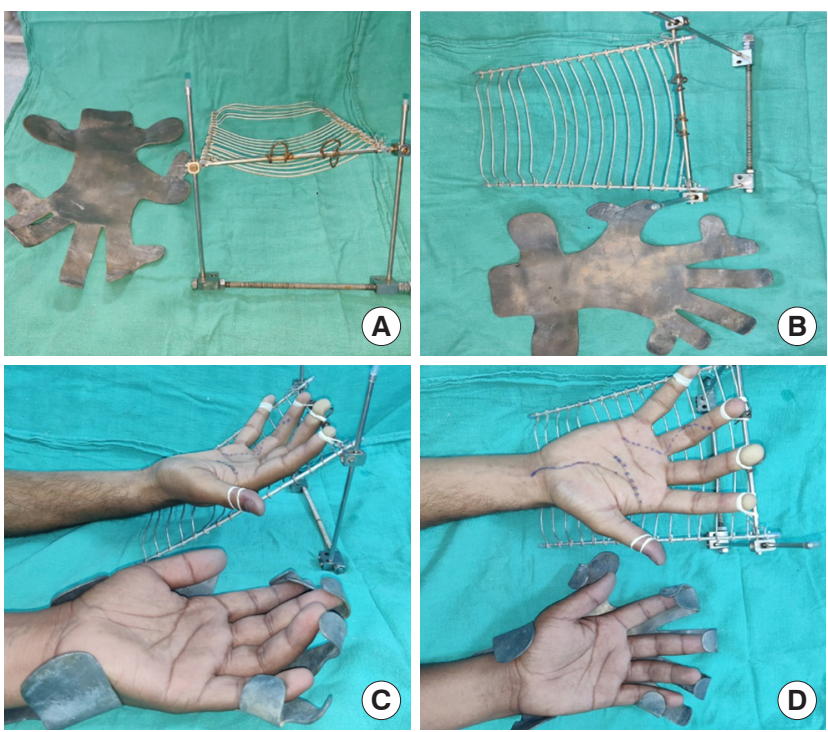

Fig. 4. Jain's hand retractor system and stand compared with a lead hand. (A) Front view. (B) Top view comparing their length. (C) Side view showing elevation advantage in Jain's hand retractor system and stand. (D) Retraction mechanism of the two devices.

debridement, for which the hand needs to be disengaged from the retractor and put in a sponge bowl to prevent spillage from cleaning. Simultaneous debridement and cleaning are not possible with currently available retractor systems or lead hands. The lead hand itself is a bulky and heavy device, weighing approximately 700-1,000 g (Fig. 4). In comparison with a conventional lead hand or other hand retractor systems, Jain's retractor system and stand has the following advantages: (1) the stand height and inclination are adjustable according to the user's need for elevation; (2) the stand can be folded or dissembled and placed in a small box; (3) the stand can be sterilized using an autoclave; (4) this system is lightweight compared to a lead hand or con- 

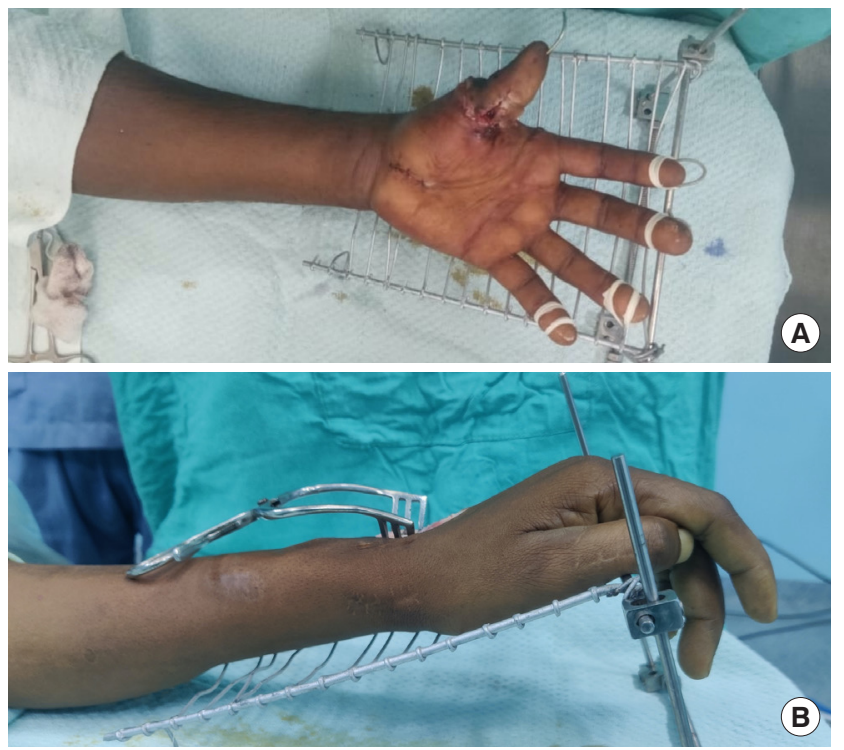

Fig. 5. Hand surgery is performed using Jain's hand retractor system and stand. (A) Palmar side. (B) Dorsal side.

ventional sandbag (used for hand elevation/positioning), which destabilizes the hand table with its weight (Fig. 4); (5) the hooks made at the ends of the stand help to keep fingers and skin flaps retracted using elastic bands or sutures and act as selfretaining retractors (Fig. 5); (6) the upward curve modification at the start of the stand provides an anatomical benefit for wrist surgery and provides better exposure; or (7) the kidney tray can be put below the stand and proper lavage can be done along with debridement without spillage of water and blood.

\section{DISCUSSION}

Hand injuries are commonly encountered in polytrauma cases [7]. There is a need for a good hand retractor system that can fulfill the needs of solo hand surgery. As hand surgery is most often performed under regional anesthesia and with few trained assistants, there is a dire need for an ergonomically efficient and economically viable retractor system. This hand retractor system and stand not only addresses the problems of elevation, positioning, and retraction, but is also reproducible and affordable. In comparison with the conventionally used lead hand, this hand retractor system and stand is lighter and less bulky (Fig. 4). Because of its simple design, any trainee or hand surgeon can easily make Jain's multipurpose hand retractor system and stand, also can modify it according to their needs.

\section{NOTES}

\section{Conflict of interest}

No potential conflict of interest relevant to this article was reported.

\section{Ethical approval}

The study was performed in accordance with the principles of the Declaration of Helsinki.

\section{Patient consent}

The patient provided written informed consent for the publication and the use of his images.

\section{ORCID}

Aakansh Jain

https://orcid.org/0000-0002-7776-7424

\section{REFERENCES}

1. Bell MS, Reitsma BJ. Solo hand surgery. Can J Plast Surg 2005; 13:145-7.

2. Bolton Surgical Ltd. Surgical instruments: lead hands [Internet]. Sheffield: Bolton Surgical Ltd.; c2021 [cited 2021 Mar 7]. Available from: https://www.boltons.co.uk/productsand-services/surgical-instruments/plastic-surgery/leadhands/lead-hands-898.

3. Hand Surgery Stand. HandStand(TM): The First Sustainable Hand Surgery Table. [Internet]. Metairie, LA: Hand Surgery Stand; c2021 [cited 2021 Mar 7]. Available from: https://www.handsurgerystand.com.

4. Madison JB, Casale AJ, Bosworth L, et al. A self-retaining retractor for hand surgery. Plast Reconstr Surg 1982;70:760-1.

5. Arem AJ. Self-retaining weighted retractors for hand surgery. Ann Plast Surg 1980;5:326-7.

6. Doi H, Ogawa Y. A new malleable self-retaining retractor. Ann Plast Surg 1997;38:543-5.

7. Singh SB, Vardhan H, Halageri S, et al. Demographic profile of hand injuries in North India: a tertiary care hospital experience. Albanian J Trauma Emerg Surg 2021;5:759-63. 